\title{
Epidermolysis Bullosa Acquisita Antigen Is the Globular Carboxyl Terminus of Type VII Procollagen
}

\author{
David T. Woodley, ${ }^{*}$ Robert E. Burgeson, ${ }^{\ddagger}$ Greg Lunstrum, ${ }^{\ddagger}$ Leena Bruckner-Tuderman, \\ Melinda J. Reese, ${ }^{*}$ and Robert A. Briggaman* \\ ${ }^{*}$ Department of Dermatology, University of North Carolina Medical School, Chapel Hill, North Carolina $27514 ;{ }^{\ddagger}$ Department of \\ Biochemistry, Cell Biology and Anatomy and Dermatology, Shriners Hospital for Crippled Children, Oregon Health Sciences \\ University, Portland, Oregon 97201; and ${ }^{\S}$ Department of Dermatology, University of Zurich, Zurich, Switzerland 8091
}

\begin{abstract}
Epidermolysis bullosa acquisita (EBA) is a severe, chronic blistering disease of the skin. EBA patients have circulating and tissue-bound autoantibodies to a large $\left(M_{\mathrm{r}}=290,000\right)$ macromolecule that is localized within the basement membrane zone between the epidermis and dermis of skin, the site of blister formation. The "EBA antigen" is known to be distinct from laminin, heparan sulfate proteoglycan, fibronectin, the bullous pemphigoid antigen, elastin, and collagen types I, II, III, IV, and V. Sera from patients with EBA, two monoclonal antibodies to the EBA antigen, and a monoclonal antibody to the carboxyl terminus of type VII procollagen identically label human amnion and skin by immunofluorescent and immunoelectron microscopy. Western immunoblots of the EBA antigen extracted from skin and of type VII procollagen labeled with the above sera and antibodies are identical. None of the sera or antibodies labels Western blots of pepsinized type VII collagen which is missing the globular amino and carboxyl terminal domains. These data show that the EBA antigen is the carboxyl terminus of type VII procollagen.
\end{abstract}

\section{Introduction}

Epidermolysis bullosa acquisita (EBA) ${ }^{1}$ is an acquired subepidermal blistering disease of the skin that was first described in 1896 (1). In 1972, Roenigk et al. (2) reviewed the world literature on EBA and developed formal criteria for the diagnosis. These patients have tissue-bound and serum autoantibodies (3-5) against a $290,000-\mathrm{D}$ matrix molecule that is localized within the basement membrane zone (BMZ) that separates the epidermis from the dermis (6). Since this matrix component was first defined by EBA autoantibodies, it was called the "EBA antigen" (6). Nevertheless, the EBA antigen is a normal constituent of all basement membranes beneath stratified squamous epithelium. The EBA antigen shares the BMZ beneath the epidermis with other large matrix components such as laminin, heparan sulfate proteoglycan, and type IV collagen (7). Previously, we have shown that the EBA antigen is a connective tissue molecule that is distinct from laminin, fibronectin, elastin, heparan sulfate proteoglycan, and collagen types I,

Address all correspondence to Dr. David T. Woodley, Department of Dermatology, University of North Carolina, Chapel Hill, NC 27514. 1987.

Received for publication 27 July 1987 and in revised form 7 October

1. Abbreviations used in this paper: $\mathrm{BMZ}$, basement membrane zone; EBA, epidermolysis bullosa acquisita.

J. Clin. Invest.

(c) The American Society for Clinical Investigation, Inc.

0021-9738/88/03/0683/05 $\$ 2.00$

Volume 81, March 1988, 683-687
IV, and V (6). Partial characterization of the EBA antigen has shown that it is composed of two similarly sized $(145,000 \mathrm{kD})$ domains, one of which is collagenase-sensitive and the other which is collagenase-resistant and rich in carbohydrates (8). In this report, we now show that the EBA antigen is type VII procollagen. $^{2}$

\section{Methods}

Materials. All chemicals came from Sigma Chemical Company, Saint Louis, MO, unless otherwise specified. All peroxidase-labeled antibodies were obtained from CooperBiomedical, Inc., Malvern, PA.

Antibodies. Antibodies from the sera of five patients with EBA were used. These sera have been shown previously to label the dermal-epidermal junction of human skin by indirect immunofluorescence, the lamina densa and sub-lamina densa regions of the basement membrane within the dermal-epidermal junction of skin by indirect immunoelectron microscopy, and the 290- and 145-kD EBA antigen chains by Western blot analysis of urea extracts of human skin basement membrane $(5,6,8)$. A new MAb, EBA-1, with properties identical to those of antibodies in the serum of patients (13) was also used and compared with a well-characterized MAb to the carboxyl terminus of type VII procollagen (14). Control reagents were five normal human sera, two sera from patients with bullous pemphigold $(6,8)$, normal mouse serum (CooperBiomedical), and monoclonal culture medium.

Indirect immunofluorescence/indirect immunoelectron microscopy. Routine indirect immunofluorescent staining $(6,13,15,16)$ was performed on fresh, frozen human skin and amnion with antibodies in working dilutions of 1:10. Each substrate was immediately obtained from either the operating room or the delivery suite, placed in OCT cryogel medium (Lab Tek, Naperville, IL), immersed in liquid nitrogen, and cut on a cryotome set at $4 \mu \mathrm{m}$. Identical experiments were performed on human skin that had been split through the BMZ by incubating the skin in $1 \mathrm{M}$ cold $\mathrm{NaCl}$ as previously described $(6,16$, 17). This procedure fractures the basement membrane through the $B M Z$ and places the lamina densa and EBA antigen with the dermalside of the separation, while placing the bullous pemphigoid antigen with the epidermal-side of the separation $(6,16,17)$.

To determine the precise ultrastructural localization within the $\mathrm{BMZ}$ to which these antibodies bind, immunoelectron microscopy was

2. Nomenclature and relative molecular weights: Type VII procollagen refers to a triple helical macromolecule of three identical alpha chains (9). Each chain contains an amino terminal globular domain $\left(M_{\mathrm{r}}\right.$ $=\sim 30,000$ when estimated using globular standards $(9,10)$, a collagen-helical domain $\left(M_{\mathrm{r}}=170,000\right.$ when estimated using collagenhelix standards or 145,000 when estimated using globular standards), and a carboxyl-terminal globular domain $\left(M_{\mathrm{r}}=145,000-150,000\right.$ when estimated using globular protein standards). The calculated $M_{\mathrm{r}}$ of a type VII procollagen alpha chain is $\sim 345,000$. When estimated using globular standards, the value is $\sim 295,000-300,000$. The term "type VII collagen" has previously been used to refer to only the triple-helical domain of the molecule $(9,11)$, but more properly should refer to the tissue form (12). The structure of the tissue form of the macromolecule is not well characterized. In this report, the portion of the chains contributing only to the collagen helix will be referred to as pepsinized type VII collagen (12). 
performed on human skin with anti-type VII procollagen and monoclonal anti-EBA antigen antibody as previously described $(5,6,13)$.

Extracellular matrix components. Urea extracts of human skin basement membrane proteins were prepared as previously described $(6,8,13)$. Alternatively, human skin BMZ proteins were prepared by slicing human skin to a 0.4-mm thickness with a keratome; incubating the skin in $0.010 \mathrm{M}$ cysteine and $1 \mathrm{M} \mathrm{NaCl}$ at $4^{\circ} \mathrm{C}$ for $72 \mathrm{~h}$; separating the epidermis from the underlying basement membrane; pulverizing the $\mathrm{BMZ}$ into a fine powder in liquid nitrogen; and extracting the powder $(4 \mathrm{~g} / 80 \mathrm{ml}$ ) with $6 \mathrm{M} \mathrm{GuHCl}, 0.025 \mathrm{M}$ Tris- $\mathrm{HCl}(\mathrm{pH} 8.0)$, 0.010 cysteine, $0.001 \mathrm{M}$ PMSF, and $0.005 \mathrm{M} N$-ethylmaleimide (guanidine extraction buffer) at $4^{\circ} \mathrm{C}$ for $14 \mathrm{~h}$. Treatment of these proteins with bacterial collagenase (Form III, Advance Biofractures, Lynbrook, NY) at $37^{\circ} \mathrm{C}$ in calcium-containing buffer yielded a partially purified preparation of the $145-\mathrm{kD}$ collagenase-insensitive EBA domain as previously reported (8). Type VII procollagen from a human epidermal WISH cell line, ATCC-CCL-25 (American Type Culture Collection, Rockville, MD) was prepared according to Lunstrum et al. (9). Pepsinized type VII collagen extracted from human amnion was prepared according to Bentz et al. (11).

Whole human amnion and human skin basement membrane were pulvarized in liquid nitrogen into a fine powder, lyophilized, and weighed. $4 \mathrm{~g}$ of each material was incubated at $4^{\circ} \mathrm{C}$ in guanidine extraction buffer with constant stirring for $14 \mathrm{~h}$ and then centrifuged at $100,000 \mathrm{~g}$ for $1 \mathrm{~h}$. The supernatant containing the extracted proteins and the pellets were dialyzed against water and then lyophilized. The lyophilized materials were then solubilized in $0.01 \mathrm{M} \mathrm{NaPO}_{4}, 2 \% \mathrm{SDS}$, pH 6.8 (sample buffer), at a concentration of $5 \mathrm{mg} / \mathrm{ml}$, and subjected to SDS-PAGE.

SDS-PAGE and western immunoblots. Proteins were subjected to SDS-PAGE according to Laemmli (18) using 5\% polyacrylamide slab gels and then Western immunoblotting as previously described $(6,13$, $19,20)$. The proteins separated by SDS-PAGE and transferred to nitrocellulose paper were then reacted with primary antibodies. Specific binding of a primary antibody to one or more of the transferred proteins was detected by the reaction of a secondary peroxidase-labeled antibody to the primary antibody and 4-chloro-napthol substrate that creates a blue band in the presence of peroxidase $(13,14,17)$.

In addition to one-dimensional SDS-PAGE, skin BMZ proteins and type VII procollagen were subjected to two-dimensional gel electrophoresis $(21)$ and Western immunoblotting $(22,23)$ against antibodies to type VII procollagen or the EBA antigen. This procedure allowed for a direct comparison of the isoelectric points and molecular sizes of the molecules labeled by each antibody.

\section{Results}

Indirect immunofluorescent labeling of human skin and amnion with polyclonal antibodies from EBA patients $(n=5)$, MAb to the EBA antigen (13), and MAb to type VII procollagen (14) gave identical staining. All of the antibodies labeled the BMZ beneath the epithelium of the two tissues and did not label the basement membranes surrounding blood vessels (Fig. 1). On salt-separated skin substrate, all of the antibodies stained the BMZ on the dermal-side of the separation and did not label the epidermal side. Naive observers $(n=3)$ were unable to distinguish these antibodies by their staining patterns on the three substrates.

Indirect immunoelectron microscopy of human skin with MAbs to the EBA antigen (13) and the carboxyl terminus of type VII procollagen (14) showed identical labeling of the $\mathrm{BMZ}$ with specific labeling of the lamina densa region while the lamina lucida space and sub-lamina densa regions remained unlabeled (Fig. 2).

Western immunoblots of type VII procollagen and skin basement membrane proteins with and without degradation by bacterial collagenase were labeled identically by sera from patients with EBA $(n=5)$, by MAb to the EBA antigen, and by

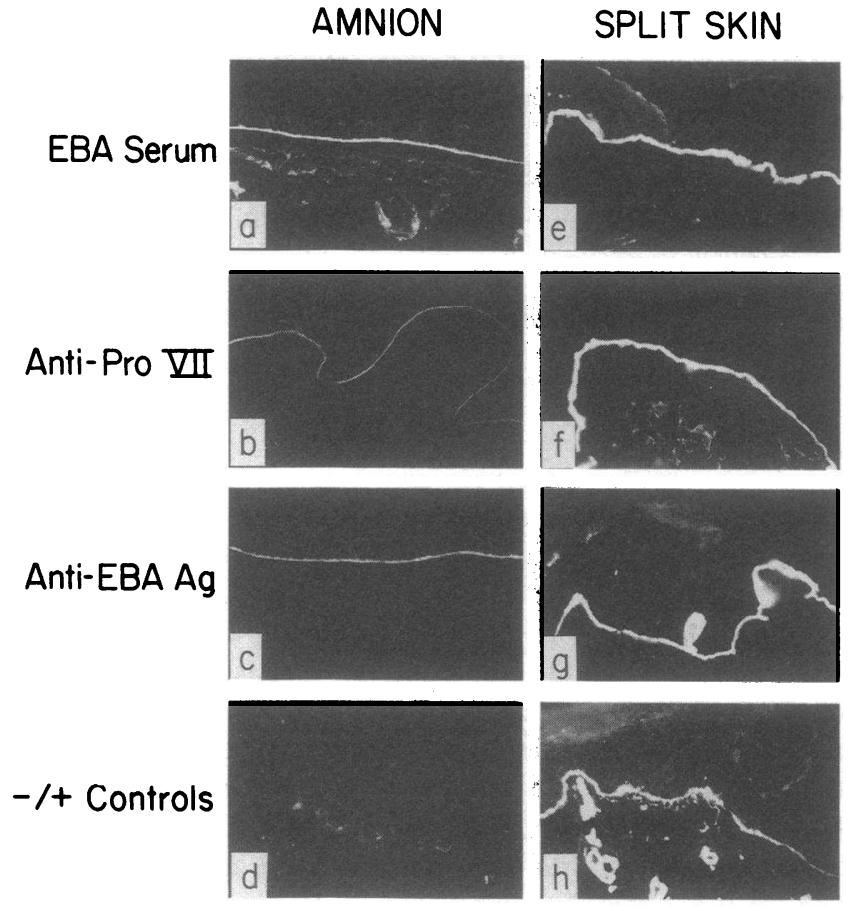

Figure 1. Indirect immunofluorescent staining of amnion $(a-d)$ or salt split human skin $(e-h)$ with serum from a patient with EBA ( $a$ and $e$ ), an MAb to type VII procollagen ( $b$ and $f$ ), an MAb to the EBA antigen ( $c$ and $g$ ), normal mouse serum $(d)$, or an MAb to type IV collagen $(h)$.

MAb to the carboxyl terminus of type VII procollagen (Fig. 3). Although each serum and antibody identically recognized a given substrate (with and without collagenase treatment), there were slight differences in the apparent molecular weights of the labeled proteins. For example, either collagenase-treated or untreated skin basement membrane proteins always demonstrated higher electrophoretic mobility than the parallel type VII procollagen derived from WISH cell cultures (Fig. 3). None of the sera or antibodies labeled type VII collagen that was extracted by pepsinization of human amnion (Fig. 4). It is known that pepsinization cuts off the globular amino and carboxyl termini from type VII procollagen and leaves a smaller molecule containing only the triple-helix (type VII collagen). This suggests that the EBA antigen and the globular carboxyl terminus of type VII procollagen are identical.

Two-dimensional gel electrophoresis of the skin BMZ proteins and type VII procollagen run in parallel and followed by Western immunoblotting simultaneously with antibodies to type VII procollagen or to the EBA antigen showed that either type of antibody labeled a band in the skin BMZ extract that had an identical pI to the labeled type VII procollagen (Fig. 5). The apparent pI of the bands labeled with antibody is 8.15. The molecular sizes of the labeled bands in the second dimension $(290 \mathrm{kD})$ are similar but not identical. As noted with SDS-PAGE and blotting, the two-dimensional gels also show that the type VII procollagen that was derived from tissue culture runs as a closely set doublet while the labeled band in the skin BMZ extract is a singlet (Fig. 5). Both bands of the closely set doublet have identical pIs and separate only slightly in the second dimension. It is likely that the small lower band in the doublet represents a degradation product from the more prominent band that occurs due to nonspecific proteolysis during the preparation of type VII procollagen from cultures of WISH cells. We have noted similar doublets in some ex- 

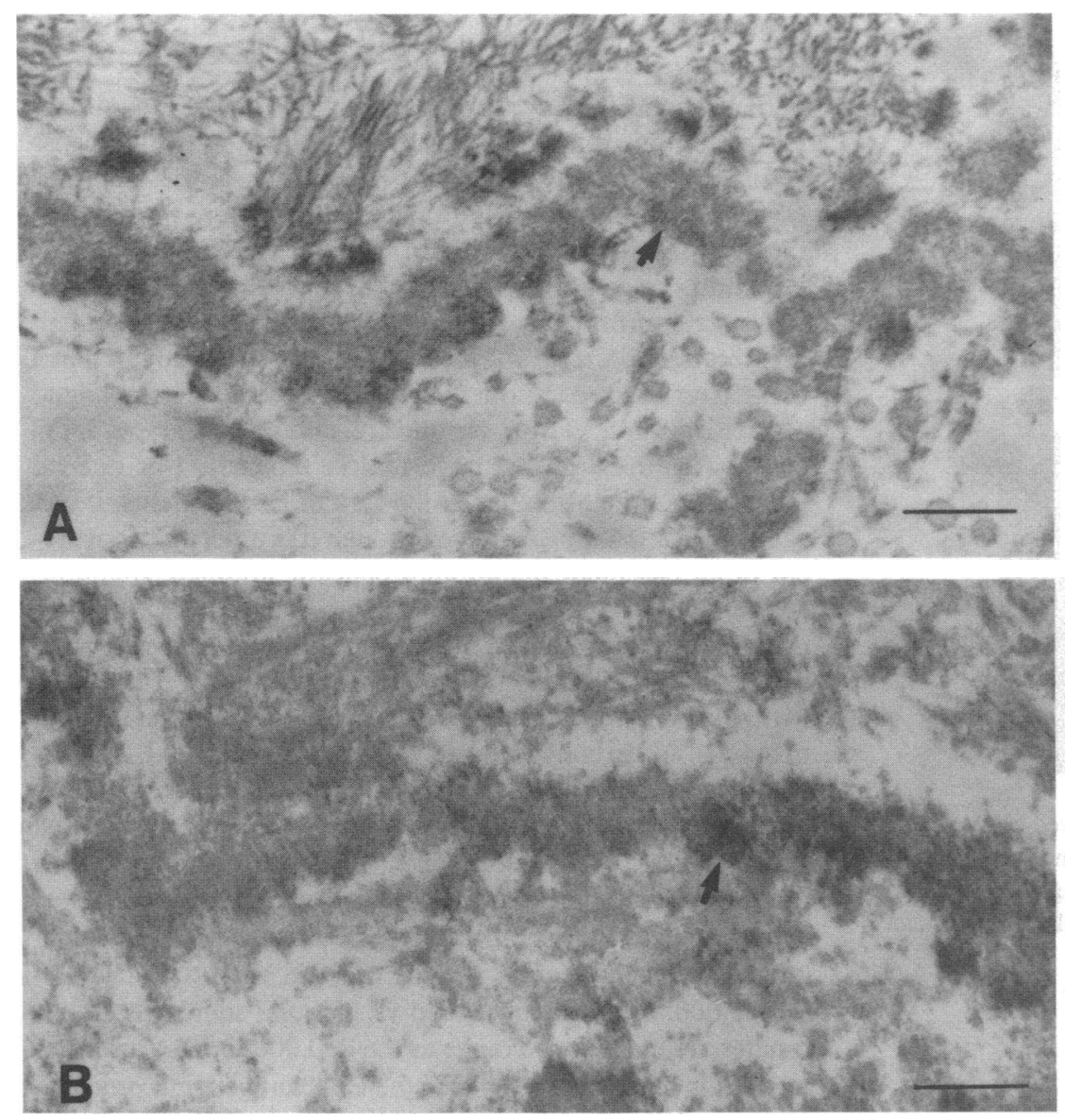

Figure 2. Immunoelectron micrographs of normal human skin using MAbs to the EBA antigen $(A)$ and type VII procollagen $(B)$. Immune deposits are present over the lamina densa and in the reticular layers (arrows). The same ultrastructural localization is seen with both antibodies. Bar, $0.25 \mu \mathrm{m}$. tracts of skin basement membrane when stringent conditions and a protease-free environment are not strictly maintained.

On a dry weight basis, more immunologically identifiable EBA antigen-type VII procollagen appeared to be extracted and labeled by a specific antibody in Western blots of skin extracts than those from amnion prepared identically (Fig. 6). $50 \mu \mathrm{g}$ of each extract were subjected to immunoblotting against identical amounts of sera (1:800 dilution) or MAbs. Under these conditions, the intensity of the labeled band was always greater in lanes containing skin basement membrane proteins. Since no labeling occurred in the nonextractable, pellet proteins from amnion or skin basement membrane, it

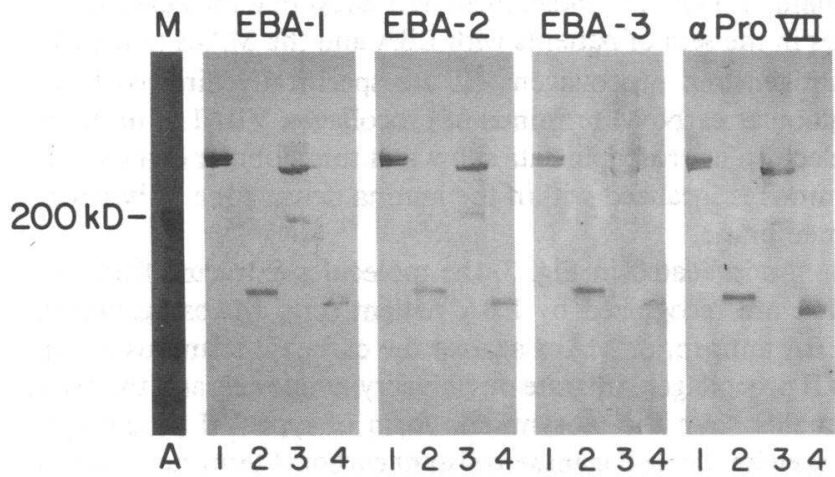

Figure 3. Western immunoblots of type VII procollagen (lanes 1 and 2) and human skin basement membrane proteins (lanes 3 and 4 ), without (lanes 1 and 3) and with (lanes 2 and 4 ) collagenase treatment. They were labeled with antibodies to type VII procollagen ( $\alpha$ Pro VII), MAb to the EBA antigens (EBA-1), and sera from two patients with EBA (EBA-2 and $E B A-3)$. appears that these methods maximally extract the immunologically identifiable EBA antigen-type VII procollagen from these two tissues (Fig. 6). Western immunoblotting is semiquantitative at best, and there is no information to indicate that the intensity of labeled bands follows Beer's law (e.g., twice the intensity equates with twice the amount of labeled protein). Nevertheless, assuming that antibody affinities are the same for EBA antigen derived from different tissues, these data may suggest that on a dry weight basis there is more EBA antigen in skin basement membranes than those in amnion.

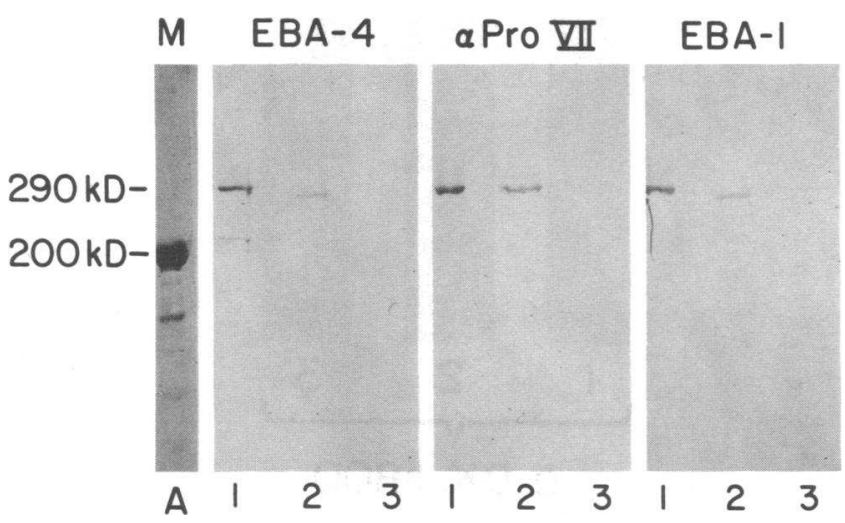

Figure 4. Identical Western immunoblots of guanidine extracts of human skin basement membrane (lane 1) or amnion (lane 2) and type VII collagen extracted from amnion by pepsinization (lane 3 ). They were labeled with antibodies from the serum of an EBA patient $(E B A-4)$, antibody to the carboxyl terminus of type VII procollagen, ( $\alpha$ Pro VII), or an MAb to the EBA antigens $(E B A-1)$. Lane $A-M$ is a high molecular weight marker stained with amido black. 


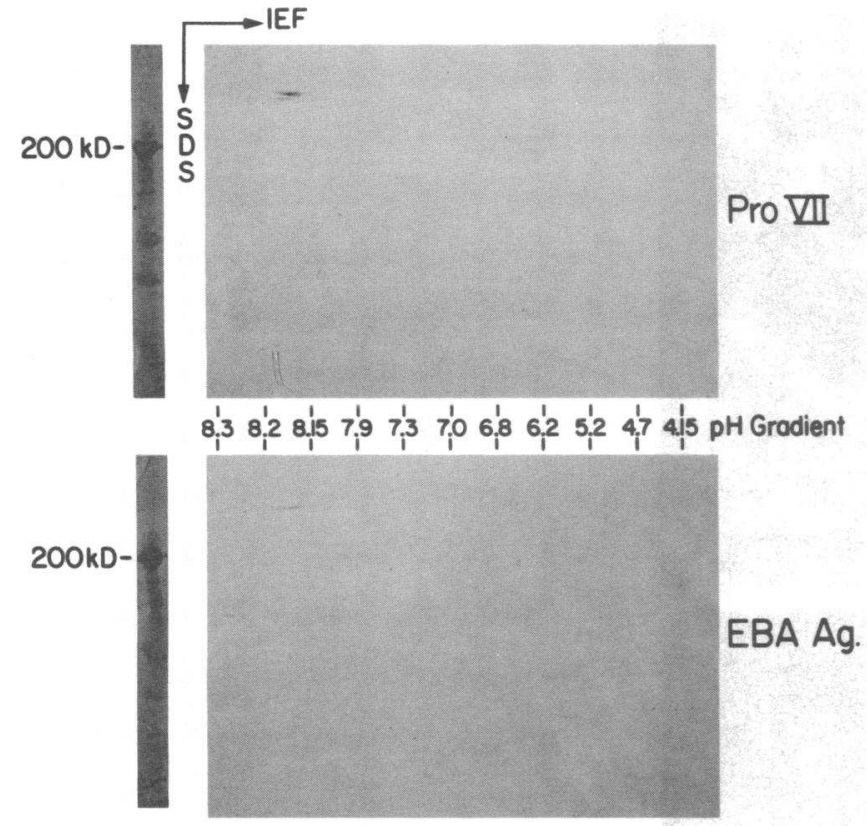

Figure 5. Two-dimensional gel electrophoresis followed by Western immunoblotting of type VII procollagen (Pro VII) and the EBA antigen $(E B A A g)$ labeled with MAb to type VII procollagen. Blotted high molecular weight markers stained with amido black are parallel to the left-hand edge of each blot.

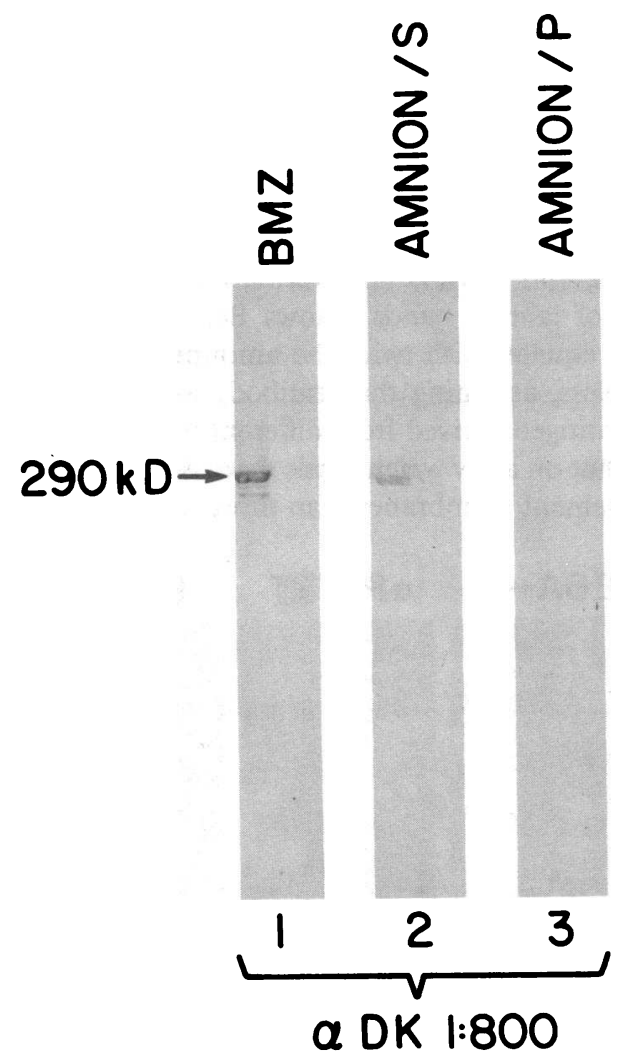

Figure 6. Western immunoblots of human skin basement membrane zone proteins (lane 1 ) and human amnion proteins (lane 2) extracted with equal volumes of guanidine hydrochloride extraction buffer (see Methods) per unit of dry weight of tissue. The residual pellet proteins left after the amnion extraction are shown in lane 3. $50 \mu \mathrm{g}$ of each substrate was electrophoresed on a 5\% SDS-polyacrylamide slab gel, blotted, and labeled with a 1:800 dilution of serum from an EBA patient.

\section{Discussion}

Patients with EBA have serum and tissue-bound autoantibodies that bind within and immediately below the lamina densa region of the cutaneous BMZ (4-6), which is the exact site where blister formation is initiated. Previously, we reported that the molecule within the BMZ to which these autoantibodies bind is recognized as a 290,000-D protein in Western immunoblots of proteins extracted from human skin basement membrane and that this molecule is distinct from other known connective tissue components such as the bullous pemphigoid antigen, laminin, elastin, fibronectin, and collagen types I, IV, and $V(6)$. Because this large matrix molecule was defined initially by autoantibodies in the sera of EBA patients, it was called the "EBA antigen" even though it is a normal constituent of all basement membranes beneath stratified squamous epithelium.

It was shown that the 290,000-D EBA antigen was composed of a 145,000-D collagenous domain and a 145,000-D (when calibrated vs. globular standards) noncollagenous domain rich in carbohydrates $(8,24)$. Using both MAb and patient's sera, native 290-kD EBA antigen was immunoprecipitated from human keratinocyte cultures (25). Independently, Bentz et al. (11) and colleagues $(9,14,26)$ purified a new unique collagen, termed type VII collagen, that is associated with anchoring fibrils in basement membranes (9). The calculated relative molecular weight of type VII procollagen is $\sim 345,000$, but when measured vs. globular standards, $M_{\mathrm{r}}$ estimates are in the same range as the 290,000-D EBA antigen. ${ }^{2}$

In this report, we show that the EBA antigen and procollagen type VII are the same. MAbs to type VII procollagen specifically label the EBA antigen in protein extracts of skin basement membrane. Conversely, antibodies in the sera of EBA patients and an MAb to the EBA antigen show labeling of type VII procollagen identical to the labeling with an MAb to procollagen VII. Regardless of whether the antibodies are to the EBA antigen or to procollagen VII, the labeling in Western blots of untreated or collagenase-treated procollagen VII or of proteins extracted from skin basement membrane is identical. None of the sera or antibodies recognizes pepsinized type VII collagen extracted from human amnion which is known to lack the globular amino and carboxyl termini. In addition, none of the patient sera identifies peptides in the $30-\mathrm{kD}$ size range that corresponds to the amino terminal globular domain. Taken as a whole, these data show that the autoantibodies in the sera of patients with EBA and the MAbs to the EBA antigen and procollagen VII are specifically directed to the globular carboxyl terminus of procollagen VII. The immunoelectron microscopic data show that this globular carboxyl terminus is localized within the lamina densa zone of basement membrane.

As indicated in Fig. 3, the molecules extracted from skin that are recognized by EBA patient sera, MAbs against the EBA antigen, or MAbs against the carboxyl terminus of type VII procollagen, migrate on polyacrylamide gels as if they were smaller than the biosynthetic form of type VII procollagen. Likewise, the collagenase-resistant carboxyl terminus from the extracted molecule is also apparently smaller. The significance of this observation is unclear. Recent evidence (10) suggests that the tissue form of type VII collagen lacks the amino-terminal globular domain, but retains the intact carboxyl-terminal domain. In view of these data, it is likely that the apparent smaller size of the extracted EBA antigen (type VII procolla- 
gen) is due to physiological removal of the amino terminus and to modification of the carboxyl terminus.

It has been shown that collagens are capable of having specific affinity to other matrix molecules such as fibronectin, laminin, and heparan sulfate proteoglycan (27-30). It is thought that these interactions may be important for the integrity of basement membranes and in maintaining epidermaldermal adherence. Recently, we have shown that the EBA antigen, like most collagens, has marked affinity for fibronectin, another major matrix component of skin that is concentrated near the BMZ (31). The skin blisters in EBA often occur in the absence of clinical or histological inflammation (1). It is conceivable that the fracturing of the epidermal-dermal junction in EBA may not be due to an inflammatory process but rather to a direct abrogation of affinities between the type VII procollagen and other necessary matrix molecules (such as fibronectin) by autoantibody deposition at this site.

\section{Acknowledgments}

This work was supported by grants AM 33625 (Dr. Woodley) and AR-10546 (Dr. Briggaman) from the National Institutes of Health, and grant IN-15Y from the American Cancer Society. Dr. Woodley is a recipient of Research Career Development Award AM-01540.

The authors are grateful to Ms. Donna Foushee for typing this manuscript.

\section{References}

1. Elliot, G. T. 1865. Two cases of epidermolysis bullosa. J. Cutan. Genitourin. Dis. 13:10-18.

2. Roenigk, H. H. Jr., J. G. Ryan, and W. F. Bergfeld. 1971. Epidermolysis bullosa acquisita: report of three cases and review of the literature. Arch. Dermatol. 103:1-10.

3. Gibbs, R. H., and H. R. Minus. 1975. Epidermolysis bullosa acquisita with electron microscopic studies. Arch. Dermatol. 111:215220.

4. Nieboer, C., D. M. Boorsma, M. J. Woerdeman, and G. L. Kalsbeek. 1980. Epidermolysis bullosa acquisita: immunofluorescence, electron microscopic and immunoelectron microscopic studies in four patients. Br. J. Dermatol. 102:383-392.

5. Yaoita, H., R. A. Briggaman, T. J. Lawley, T. T. Provost, and S. I. Katz. 1981. Epidermolysis bullosa acquisita: ultrastructural and immunological studies. J. Invest. Dermatol. 76:288-292.

6. Woodley, D. T., R. A. Briggaman, E. J. O'Keefe, A. O. Inman, L. L. Queen, and W. R. Gammon. 1984. Identification of the skin basement-membrane autoantigen in epidermolysis bullosa acquisita. N. Engl. J. Med. 310:1007-1013.

7. Stanley, J. R., D. T. Woodley, S. I. Katz, and G. R. Martin. 1982. Structure and function of basement membrane. J. Invest. Dermatol. 79(Suppl. 1):69s-72s.

8. Woodley, D. T., E. J. O'Keefe, M. J. Reese, G. H. Mechanic, R. A. Briggaman, and W. R. Gammon. 1986. The epidermolysis bullosa acquisita antigen, a major component of the cutaneous basement membrane is a glycoprotein with collagenous domains. J. Invest. Dermatol. 86:668-672.

9. Lunstrum, G. P., L. Y. Sakai, D. R. Keene, N. P. Morris, and R. E. Burgeson. 1986. Large complex globular domains of Type VII procollagen contribute to the structure of anchoring fibrils. J. Biol. Chem. 261:9042-9048.

10. Lunstrum, G. P., H.-J. Kuo, L. M. Rosenbaum, D. R. Keene, R. W. Glanville, L. Y. Sakai, and R. E. Burgeson. 1987. Anchoring fibrils contain the carboxyl-terminal globular domain of type VII procollagen, but lack the amino-terminal globular domain. J. Biol. Chem. 262:13706-13712.

11. Bentz, H., N. P. Morris, L. W. Murray, L. Y. Sakai, D. W. Hollister, and R. E. Burgeson. 1983. Isolation and partial characterization of a new human collagen with an extended triple-helical structural domain. Proc. Natl. Acad. Sci. USA. 80:3168-3172.
12. Bruckner-Tuderman, L., U. W. Schnyder, K. H. Winterhalter, P. Bruckner. 1987. Tissue form of type VII collagen from human skin and dermal fibroblasts in culture. Eur. J. Biochem. 165:607-611.

13. Paller, A. S., L. L. Queen, D. T. Woodley, E. J. O'Keefe, W. R. Gammon, and R. A. Briggaman. 1985. A mouse monoclonal antibody against a newly discovered basement membrane component, the epidermolysis bullosa acquisita antigen. J. Invest. Dermatol. 84:215-217.

14. Hessle, H., L. Y. Sakai, D. W. Hollister, R. E. Burgeson, and E. Engvall. 1984. Basement membrane diversity detected by monoclonal antibodies. Differentiation. 26:49-54.

15. Beutner, E. H., R. H. Nisengard, and V. Kumar. 1979. In Immunopathology of the Skin. 2nd ed. E. H. Beutner, T. P. Chorzelski, and S. F. Bean, editor. John Wiley \& Sons, Inc., New York. 29-76.

16. Woodley, D. T., D. Sauder, M. J. Talley, M. Silver, G. Grotendorst, and E. Qwarnstrom. 1983. Localization of basement membrane components after dermal-epidermal junction separtion. J. Invest. Dermatol. 681:149-152.

17. Gammon, W. R., R. A. Briggaman, A. O. Inman, L. L. Queen, and C. E. Wheeler, Jr. 1984. Differentiating anti-laminin lucida and anti-sublamina densa anti-BMZ antibodies by indirect immunofluorescence on $1.0 \mathrm{M}$ sodium chloride-separated skin. J. Invest. Dermatol. 82:139-144.

18. Laemmli, U. K. 1970. Cleavage of structural proteins during the assembly of the head of bacteriophage T4. Nature (Lond.). 227:680-685.

19. Burnette, W. N. 1981. "Western blotting": electrophoretic transfer of proteins from polyacrylamide gels to unmodified nitrocellulose and radiographic detection with antibody and radioiodinated protein A. Anal. Biochem. 112:195-203.

20. Towbin, H. T., T. Straehelin, and J. Gordon. 1979. Electrophoretic transfer of proteins from polyacrylamide gels to nitrocellulose sheets: procedures and some applications. Proc. Natl. Acad. Sci. USA. 76:4350-4354.

21. O'Farrell, P. H. 1975. High resolution two-dimensional electrophoresis of proteins. J. Biol. Chem. 250:4007-4021.

22. Koulu, L., A. Kusumi, M. S. Steinberg, V. Klaus-Kovtun, and J. R. Stanley. 1984. Human autoantibodies against a desmosomal core protein in pemphigus foliaceus. J. Exp. Med. 160:1509-1519.

23. Woodley, D. T., V. J. Scheidt, M. J. Reese, A. S. Paller, T. O. Manning, T. Yoshiike, and R. A. Briggaman. 1987. Localization of the alpha $3(\mathrm{~V})$ chain of type $\mathrm{V}$ collagen in human skin. J. Invest. Dermatol. 88:246-252.

24. Yoshiike, T., D. T. Woodley, and R. A. Briggaman. Epidermolysis bullosa acquisita antigen: relationship between the collagenasesensitive and -resistant domains. J. Invest. Dermatol. In press.

25. Woodley, D. T., R. A. Briggaman, W. R. Gammon, and E. J. O'Keefe. 1985. The epidermolysis bullosa acquisita antigen is synthesized by human keratinocytes cultured in serum-free medium. Biochem. Biophys. Res. Commun. 130:1267-1272.

26. Burgeson, R. E., N. P. Morris, L. W. Murray, K. G. Duncan, D. R. Keene, and L. Y. Sakai. 1986. The structure of type VII collagen. Ann. NY Acad. Sci. 460:47-57.

27. Del Rosso, M., R. Capelletti, M. Viti, S. Vannucchi, and V. Chiargui. 1981. Binding of basement membrane glycoprotein laminin to glycosaminoglycans. Biochem. J. 199:699-704.

28. Sakahita, S., E. Engvall, and E. Ruoslahti. 1980. Basement membrane glycoprotein laminin binds to heparin. FEBS (Fed. Eur. Biochem. Soc.) Lett. 116:243-246.

29. Terranova, V. P., C. N. Rao, T. Kalebec, I. M. Marguiles, and L. A. Liotta. 1983. Role of laminin in attachment of PAM 212 (epithelial) cells to basement membrane. Proc. Natl. Acad. Sci. USA. 80:444448.

30. Woodley, D. T., C. N. Rao, J. R. Hassell, L. A. Liotta, and G. R. Martin. 1983. Interactions of basement membrane components. Biochim. Biophys. Acta. 761:278-283.

31. Woodley, D. T., E. J. O'Keefe, J. A. McDonald, M. J. Reese, R. A. Briggaman, and W. R. Gammon. 1987. Specific affinity between fibronectin and the epidermolysis bullosa acquisita antigen. J. Clin. Invest. 79:1826-1830. 